\title{
Microinstability of the hip: a previously unrecognized pathology
}

\author{
Ioanna Bolia ${ }^{1}$ \\ Jorge Chahla ${ }^{1}$ \\ Renato Locks ${ }^{1}$ \\ Karen Briggs ${ }^{1}$ \\ Marc J. Philippon ${ }^{1,2}$ \\ 1 Steadman Philippon Research Institute, Colorado, USA \\ 2 The Steadman Clinic, Colorado, USA
}

Corresponding author:

Marc J. Philippon, MD

Steadman Philippon Research Institute

The Steadman Clinic

181 West Meadow Drive, Suite 400

Vail, Colorado 81657, USA

E-mail: drphilippon@sprivail.org

\section{Summary}

Background: Hip microinstability is an established diagnosis; however, its occurrence is still debated by many physicians. Diagnosis of hip microinstability is often challenging, due to a lack of specific signs or symptoms, and patients may remain undiagnosed for long periods. This may lead to early manifestation of degenerative joint disease. Consequently, careful patient and family history must be obtained and diagnostic imaging should follow. After a thorough clinical evaluation of the patient with suspected hip microinstability, the physician should focus on how to improve symptoms and functionality in daily and sports activities.

Purpose: The purpose of this review article was to give a current update regarding this diagnosis and to provide a complete diagnostic approach in order to effectively treat hip microinstability.

Methods: We reviewed the literature on the diagnosis, the non-operative and operative indications for the treatment of this complex and often misdiagnosed pathology.

Conclusion: Conservative treatment is considered the best initial approach, though, surgical intervention should be considered if symptoms persist or other hip pathology exists. Successful surgical intervention, such as hip arthroscopy, should focus on restoring the normal anatomy of the hip joint in order to regain its functionality.
The role of the hip joint capsule has gained particular research interest during the last years, and its repair or reconstruction during hip arthroscopy is considered necessary in order to avoid iatrogenic hip microinstability. Various capsular closure/plication techniques have been developed towards this direction with encouraging results.

Level of evidence: $\mathrm{V}$.

KEY WORDS: hip arthroscopy, hip microinstability, hip dysplasia.

\section{Introduction}

The native hip is a particularly constrained joint with a powerful suction seal that is imperative for optimal function of the joint ${ }^{1,2}$. The hip capsule is one of the most important static stabilizers of the hip joint ${ }^{3}$ and disruption or debridement of the capsule during hip arthroscopy is a potential contributor to postoperative iatrogenic hip instability. Therefore, hip surgeons must be thoughtful of hip capsule management as hip arthroscopic procedures are increasing exponentially ${ }^{4}$. Unlike other joints in the anatomy, hip instability is generally defined as extra-physiologic hip motion that causes pain with or without symptoms of hip joint instability ${ }^{5}$. This entity is not well defined, as no objective criteria has been proposed to characterize hip microinstability 6

The source of pain and symptoms are subtle anatomic abnormalities and in most cases are associated with a repetitive movement generally involving rotations and axial load ${ }^{6}$. Alternatively, ligamentous laxity or muscle weakness can also mimic hip microinstability $^{7}$. As a result of the altered biomechanics between the femoral head and the acetabulum, the surrounding structures such as the labrum, the capsule and the cartilage may be at risk. Symptoms related to this pathology are persistent groin pain, tendinitis from dynamic stabilizer overload or pistoning of the femoroacetabular joint.

latrogenic microinstability is a significant cause for failure of hip arthroscopy and a growing cause of revision hip arthroscopy. Therefore, awareness of this pathology is vital for the surgeon in order to avoid unnecessary insult to the capsule or the labrum during hip arthroscopy and thus impede future iatrogenic pathology. The purpose of this article was to review the most relevant data from anatomy and biomechan- 
ics for diagnosis and treatment of this pathology. This article submits to the ethical standards of the journal ${ }^{8}$.

\section{Anatomy and Biomechanics}

The bony part of the hip joint consists of the acetabulum and the femoral head that are placed together to create a "ball and socket" mechanism in order to serve the mobility and stability properties of the hip joint. As a result, the femoral head receives $170^{\circ} \mathrm{cov}$ erage from the acetabular surface that creates a quasi-hemisphere ${ }^{7}$. More specifically, its posterior coverage is greater than the anterior, due to the acetabular tilt in the coronal plane $\left(48^{\circ}\right)$ as well as in the sagittal plane $\left(21^{\circ}\right)^{9}$. The femoral neck is inclined superiorly $130^{\circ}$ relative to the femoral shaft and is also $10^{\circ}$ anteverted relative to the femoral transcondylar axis ${ }^{7}$. In order to standardize the anatomical approach to the acetabulum during the hip arthroscopy procedure, Philippon et al. established a reproducible method to arthroscopically localize the anatomical landmarks on the acetabulum ${ }^{10}$. According to their model, the 3:00 position on the clock face corresponds to the superior margin of the psoas $U$ that can be easily located as well as the stellate crease at 12 o'clock.

Apart from the bones, the stability of this joint is based on the soft tissue components that mainly include the labrum, the capsule, the static hip stabilizers (ligamentum teres and capsular ligaments) as well as the dynamic hip stabilizers (muscles).

\section{The Acetabular Labrum}

The labrum is an avascular structure made of fibrocartilage, which runs along the acetabular rim, being continuous with the transverse acetabular ligament at the site of the acetabular fossa ${ }^{11}$. In addition, a transitional zone of calcified cartilage (1-2 mm) connects the labrum with the acetabular articular cartilage ${ }^{12}$. The labrum provides stability to the hip joint by increasing the acetabular volume by $20 \%$ and the acetabular surface area by $25 \%$ approximately ${ }^{12}$.

The presence of the labrum increases the intraarticular negative hydrostatic fluid pressure contributing to the "suction cup", preventing the leak of fluid from the central compartment ${ }^{5}$. In a study of eight cadaveric hips, Philippon et al. $^{2}$ underlined the role of the acetabular labrum in the hip fluid seal by measuring the intra-articular fluid pressurization in six different hip joint states including the intact state, labral tear, labral repair (looped vs through the tissue sutures), partial and complete resection as well as the reconstruction with iliotibial band state. They reported that partial labral tears decrease the pressurization effect of the hip fluid seal and that the through-the-tissue type labral repair can achieve superior results in the restoration of fluid pressure compared with the looped type. Moreover, the same researchers studied the contribution of the labrum as well as the hip cap- sule to the distractive stability of the hip in the aforementioned six labral states ${ }^{1}$. They found that a distraction level of 1-2 mm labrum was the most important hip stabilizer, whereas in greater distraction states, the contribution of the capsule to hip stability started to increase. In addition, the partial labral tear decreased the distractive stability of the joint while the labral reconstruction procedure significantly improved it. Consequently, a relationship between labral pathology and hip microinstability seems to exist and the contribution of the capsule to the hip joint stability cannot be ignored. When loading forces are applied to the hip joint, the labrum is considered to have a protecting role by distributing those stresses ${ }^{13}$. The presence of nociceptors and proprioceptors ${ }^{14}$ in the labrum are responsible for the sensory information transferred from the hip joint to the CNS, supporting the coordination of movements that may affect the joint stability.

\section{The Hip Capsule}

The hip capsule is composed of both longitudinal and circular fibers. The longitudinal component is $60 \%$ reinforced ${ }^{5}$ owing to the existence of the three stabilization ligaments externally; the iliofemoral, the pubofemoral and the ischiofemoral ligaments. At the inner side of the capsule the circular fibers define the zona orbicularis, which encircles mainly the femoral neck, and its thickness increases while moving from the anteroinferior $(1.3 \mathrm{~mm})$ to the posterosuperior part $(4 \mathrm{~mm})^{15}$.

A pericapsular recess of the proximal hip capsule (5 $\mathrm{mm}$ proximal and medial to the acetabular rim $)^{15}$ may be an useful anatomical pearl in MRI assessment of the capsular elasticity ${ }^{16}$. Biomechanically, the capsule seems to offer in the distractional stability of the hip joint at 3-5 mm of distraction as reported by Nepple et al. $2014^{1}$.

\section{Static Hip Stabilizers}

Iliofemoral, Pubofemoral, Ischiofemoral Ligament The iliofemoral ligament, the strongest ligament of the body ${ }^{5}$, has its acetabular origin at the 1:26 position using the clock face system ${ }^{17}$. Its lateral arm follows a horizontal route along the neck of the femur to insert at the anterior greater trochanteric crest, whereas the medial arm inserts distally at the intertrochanteric line ${ }^{18}$. The ischiofemoral ligament originates at 10:15 clock position on the acetabulum ${ }^{17}$ and follows a spiral route to insert at the base of the greater trochanter ${ }^{19}$. The pubofemoral ligament arises at the 4:44 clock position on the acetabular rim ${ }^{17}$ and blends with the medial arm of iliofemoral ligament, lacking a femoral attachment site $^{5}$.

According to the contribution of the aforementioned ligaments to hip joint stability, Martin et al. hypothesized that the grade of restriction of the rotational hip movements that each of those ligaments offered, was 
alternating with the range of motion ${ }^{18}$. On a cadaveric study, they measured the contribution of the removal of each of the above hip joint ligaments to the rotational movements both in flexion and extension. For internal rotation (flexion and extension), about $60 \%$ contribution came from the ischiofemoral ligament whereas the lateral arm of the iliofemoral ligament added another $12-30 \%$. For external rotation, the greatest contribution, $50 \%$, was from the iliofemoral ligament with the pubofemoral ligament adding another $5 \%$. Importantly, the pubofemoral ligament increased its contribution to the external rotation when moving from flexion to extension where it reached its maximum contribution of $34 \%$. When all of the 3 ligaments were removed, the greatest increase in rotational (both internal and external) range of motion was seen in extension rather than the flexion. Consequently, it is assumed that the hip joint is relaxed when it is flexed, and therefore this position increases its susceptibility to injury.

\section{The Ligamentum Teres}

The ligamentum teres has a pyramidal shape with the wider part originating from the acetabular notch whereas its insertion lies into the fovea capitis of the femur (posterior and inferior to its center $)^{5}$. It's length approaches 30-35 mm but there exists great variability ${ }^{20}$ and furthermore it may not be present in $10 \%$ of individuals ${ }^{21}$.

In order to reach its maximum tightness, the hip must be flexed, adducted and externally rotated 22,23 , but according to others this occurs in flexion and abduction $^{24,25}$. Philippon et al. ${ }^{26}$ studied the structural characteristics of the ligamentum teres on 12 human cadaveric models with mean age of 53.6 years and found that the mean ultimate failure load of this ligament is $204 \mathrm{~N}$, showing similar results to another study $^{27}$. During surgery, in order to achieve $10 \mathrm{~mm}$ hip joint distraction, a traction force of 110-225 N 28 must be applied and therefore failure should not occur. The stabilizing role of this mesodermal structure has gained specific research interest. In a recent study $^{29}$, when the ligamentum teres was resected arthroscopically, both internal and external rotation of the hip was observed but other studies suggest that it is not contributing to hip stability ${ }^{30}$. Therefore, it might play a role, especially when microinstability exists in the absence of other hip joint pathology. Finally, hypertrophy of the ligamentum teres may be present in dysplastic hips or in cases of hip osteonecrosis ${ }^{30}$.

\section{Dynamic Stabilizers of the Hip Joint}

Dynamic stabilizers of the hip joint are muscles that support the hip capsule and consequently they may have an impact on hip function and stability. More specifically, the iliocapsularis muscles, the indirect head of the rectus femoris as well as the gluteus minimus, are the main representantives of this category. The iliocapsularis reinforces the anterior hip capsule especially in dysplastic hips where it is usually hypertrophied $^{31}$. Relative to their contribution to the hip capsule support, the iliocapsularis is the greatest contributor and adheres at the $2: 30$ position of the clock face, whereas the indirect head of rectus femoris attaches between 11:30 and 14:0010. Finally Walters et al. ${ }^{15}$ described the "stability arc" in the peripheral hip compartment which consists of the gluteus medius, the iliocapsularis and the superomedial head of the rectus femoris.

\section{Physical Examination}

Hip microinstability is often difficult to diagnose. The orthopedic surgeon must be relatively suspicious when assessing the patient with hip disturbances in order to come to a reliable conclusion regarding this pathology. Prior trauma to the hip joint and its mechanism of occurrence should be described in detail. Thorough personal and family history must be taken, accompanied by a comprehensive physical examination, in order to rule out non-hip joint pathology that may manifest with groin or hip pain. Such conditions include gastrointestinal, vascular, genitourinary as well as lumbosacral region issues ${ }^{32}$.

Assessment of generalized ligamentous laxity, using the Beighton's Physical Examination Criteria (Table I) ${ }^{33}$, is a component of the clinical examination that must not be neglected when looking for hip microinstability. When considering a hyperelastic patient, microinstability can only be addressed if pain or other symptoms are still present without taking into consideration

Table I. The Beighton Score.

\begin{tabular}{|c|c|c|c|}
\hline Joint/ Finding & Negative & Unilateral & Bilateral \\
\hline Passive dorsiflexion of the fifth finger $>90^{\circ}$ & 0 & 1 & 2 \\
\hline Passive flexion of thumbs to the forearm & 0 & 1 & 2 \\
\hline Hyperextension of the elbows beyond $10^{\circ}$ & 0 & 1 & 2 \\
\hline Hyperextension of the knees beyond $10^{\circ}$ & 0 & 1 & 2 \\
\hline $\begin{array}{l}\text { Forward flexion of the trunk with knees fully } \\
\text { extended and palms resting on the floor }\end{array}$ & 0 & 1 & 1 \\
\hline
\end{tabular}

Beighton Criteria: A total score of at least 5 defines hypermobility. 
the increased range of motion present on that patient $^{7}$. In addition, connective tissue disorders that can result in hyperextensible and/or unstable joints include Ehrles-Danlos syndrome, Marfan syndrome, Down syndrome and arthrochalasis multiplex congenita. Finally, sports like football, dancing, skiing and others may contribute to hip hyperextensibility and microinstability could be the result of repetitive microtrauma? ${ }^{7}$.

Pain is not always present and some patients may complain of the "hip giving away" without other symptoms ${ }^{6,34}$. Snapping, clicking, catching and popping are mechanical symptoms that may be referred from patients of that category, with or without hip impingement symptoms ${ }^{5}$. Finally, hip dysplasia issues should be carefully assessed, since, even a mild dysplasia, without additional joint pathology, can result in microinstability 5 .

Physical examination should start by inspecting the patient's posture and gait as well as the overall physical condition. Muscle atrophy or hypertrophy may alter the stabilization forces acting on the hip joint and affect its motion and stability. Active and passive range of motion of the hip joint should be evaluated and compared against the unaffected side. Finally, neurovascular examination should take place in both legs $^{35}$. Specific tests might help the physician disclose a microinstability issue of the hip. A positive hip dial test indicates increased capsular laxity. With the patient in supine position and starting from a neutral extended position, the physician internally rotates the involved limb and then releases the force. External rotation greater than $45^{\circ}$ on the axial plain in the absence of an end point defines a positive test result. While performing the dial test ${ }^{36}$ one must take into account the degree of the femoral neck anteversion of the patient, since a decreased femoral neck anteversion will increase the external rotation and give a false positive result ${ }^{37}$. In addition, a positive relocation test can be seen ${ }^{35}$. Apprehension signs during certain hip joint movements, especially external rotation, could indicate a stability problem ${ }^{38}$. Finally, when traction forces are applied, easy distraction of the hip joint shows microinstability and this test can be performed with the patient under anesthesia to confirm the diagnosis.

Relative to the evaluation of the soft tissue around the hip joint, Thomas and Ober's test should be performed to evaluate the tightness of hip flexors and iliotibial band, respectively ${ }^{38}$. An useful clinical sign that could raise a physician's suspicion for microinstability existence is a reactive spasm of the secondary stabilizing muscles around the hip joint that may be tender on palpation. This occurs in order to better stabilize the affected leg. Weakness can also be seen in those muscles due to the overwork ${ }^{38}$.

\section{Imaging}

Imaging studies contribute significantly in the diagnostic process of hip microinstability, especially in pa- tients presenting with subtle symptoms and a noncontributory physical exam that did not yield a specific conclusion.

In order to evaluate multiple joint morphological parameters $^{39}$ radiographic studies should include an AP pelvic view as well as additional views of the affected hip such as the AP view, the cross-table lateral, the false-profile view of Lequesne ${ }^{38}$ and sometimes the split view. Coxa profunda (Figure 1) or protrusio acetabuli are both associated with a deep socket. A positive cross sign (Figure 2) indicates a retroverted acetabulum similarly to the posterior wall and/or ischial spine signs. In addition, acetabular dysplasia can be

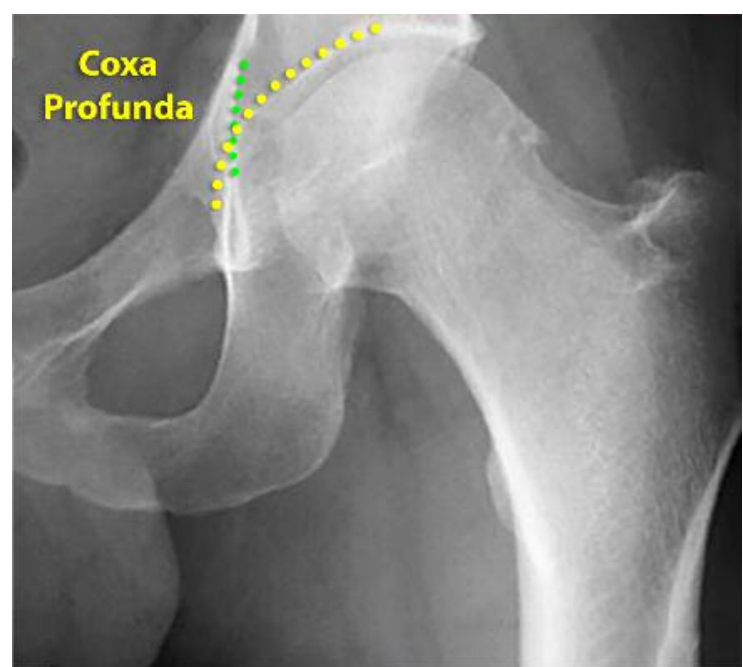

Figure 1. Anteroposterior radiographic image of a left hip demonstrating the coxa profunda sign, in which the floor of the fossa acetabuli (white dotted line) exceeds the ilioischial line medially (green dotted line).

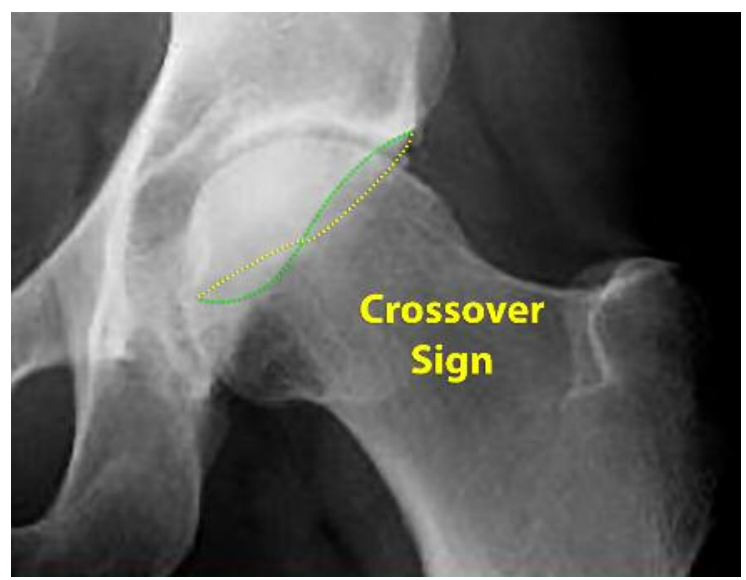

Figure 2. Anteroposterior left hip radiography showing a crossover sign. Note that the anterior rim line (yellow dotted line) lies lateral to the posterior rim (green dotted line) in the cranial aspect of the acetabulum and crossing the latter in the distal aspect of the acetabulum. 


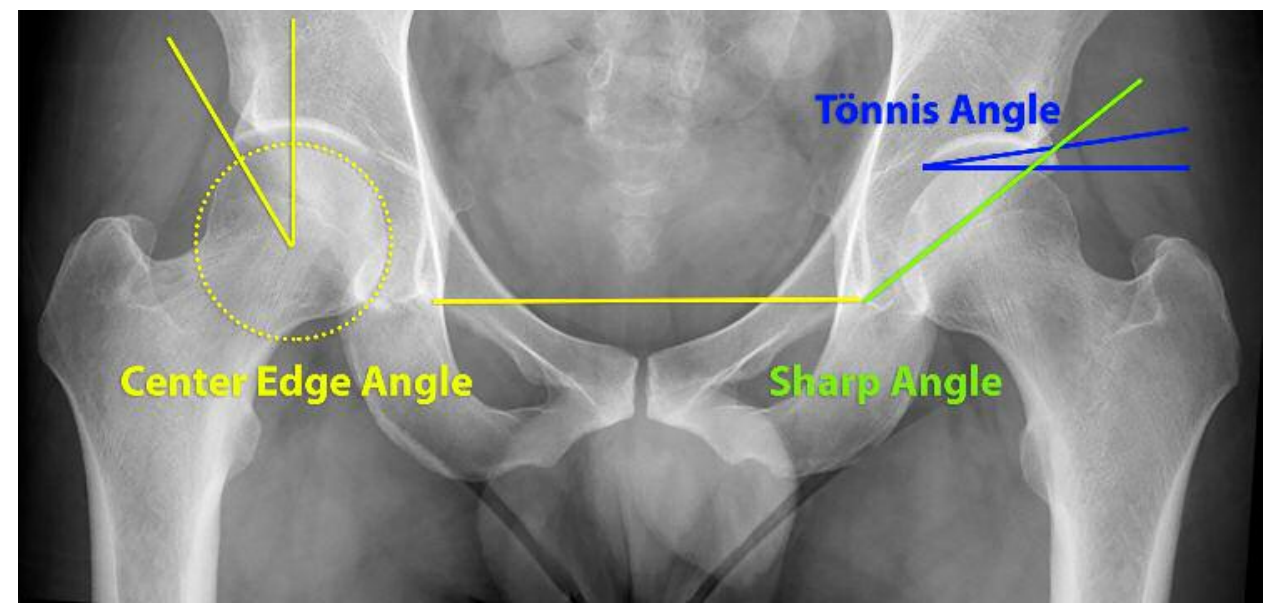

Figure 3. Anteroposterior radiographic image of both hips demonstrating the most important radiographic measurements. The lateral CEA angle is formed by a vertical line through the center of the femoral head and a line connecting the femoral head center with the most lateral edge of the acetabulum. A normal lateral center edge angle ranges between 25 to $40^{\circ}$. The Sharp angle can be measured by drawing a line that connects both tear drop signs on the right and left side of the ischial bones and measuring from this line to the most lateral aspect (most sclerotic portion) of the lateral acetabulum or the pincer deformity. Finally, the Tönnis angle that can be calculated by drawing a horizontal line connecting the base of the acetabular teardrops; next a parallel horizontal line running through the most distal point of the sclerotic acetabular sourcil; the angle is measured between this line and the lateral margin of the acetabular sourcil.

detected by measuring the lateral center-edge angle of Wiberg (less than $20-25^{\circ}$ indicates dysplasia) as well as the angle of acetabular inclination (Tönnis angle) with measurements greater than $10^{\circ}$ proving dysplastic changes (Figure 3 ). Split radiographs have gained specific interest during the last years regarding its diagnostic value in patients with hip microinstability, especially in ballet dancers ${ }^{40,41}$. Mitchell et al. ${ }^{40}$ in a study of 47 professional dancers of both genders measured the change in hip center position (HCP) on AP pelvis and AP pelvis split radiographs. They found that hip subluxation occurred at the split stance, being more prominent in women than men ${ }^{40}$. In addition, the grade of subluxation was influenced by any existent dysplastic changes, the alpha angle and the femoral neck-shaft angle. In addition, Harris et al. ${ }^{41}$ underlined the role of the split view as giving the chance to assess the hip joint when the tip of greater trochanter points at 12 o'clock of the acetabulum (internal rotation-toes forward) or at 9 o'clock (external rotation-toes up).

MRI is most useful for the detection of soft tissue abnormalities such as labral pathology, cartilage defects or capsuloligamentous deficiencies ${ }^{42,43}$. MRI arthrography, besides a more detailed view of the cartilage, offers the ability to assess the hip capsule in terms of laxity or iatrogenic injury that may result in microinstability symptoms. According to a retrospective study from Magerkurth et al. ${ }^{44}$ patients with obvious articular laxity during hip arthroscopy had a thin adjacent joint capsule $(<3 \mathrm{~mm})$ and wide anterior hip joint recess measured ( $>5 \mathrm{~mm}$ ) on MRI arthrography.

Other diagnostic modalities that can be used are CT, ultrasonography, classic arthrography or bone scans, which can be useful for addressing other pathologies when assessing patients for hip microinstability.

\section{Treatment}

In order to effectively treat hip microinstability, multiple factors should be taken into account. Although clinical and diagnostic tests are of outmost importance, the clinical presentation should rule the therapeutic algorithm where symptom relief and improvement of patient's overall functionality is the ultimate goal.

Non-surgical treatment is considered the first line approach to patients with hip microinstability symptoms. Patients should be enrolled in an activity modification module and physical therapy program. Anti-inflammatory medication should also be considered in order to alleviate pain and help any existing inflammatory process to subside quickly. Physical therapy should concentrate on strengthening the core abdominal muscles as well as the rest of the hip stabilizers, mainly the hip external rotators and the abductors. Low back muscle strengthening is also important ${ }^{45}$. In patients who followed the aforementioned conservative protocol for 6-8 weeks but failed to improve, an intra-articular injection of anesthetic to the hip joint usually follows. If significant pain improvement is seen, then surgical management may follow ${ }^{30,46}$. Also, candidates for a surgical procedure are patients with persisting instability ${ }^{30}$.

Severe hip dysplasia is an indication of an open procedure such as the acetabular and proximal femur osteotomies ${ }^{47,48}$. Hip arthroscopy has a dual role regarding the treatment of hip microinstability. The correction of borderline dysplastic characteristics, that may contribute to microinstability, in combination with the manipulation of soft tissues in and around the hip joint are responsible for the encouraging results that has already been reported in literature ${ }^{49,50}$. 
Intra-articular pathology, such as labral tears, cartilage defects and/or ligamentum teres abnormalities are often present in patients with hip microinstability. Capsular laxity and/or capsular defects also play an important role. Arthroscopic thermal capsuloraphy has been described by Philippon et al. and the subsequent shrinkage of the joint space enhanced hip stability ${ }^{46}$. A modern approach is the arthroscopic capsular plication 45,51 , where absorbable sutures are placed on the iliofemoral ligament in order to correct the capsular defect and increase its tightness, bringing together the two capsular edges. Tightness could be monitored by placing more sutures based on the degree of the desired stabilization ${ }^{38}$. Relative to the post-operative management of these patients, partial weight bearing for 4 weeks and hip brace for 6 weeks is applied. The brace should prevent external rotation, abduction and hip extension movements in order to preserve capsular integrity after repair or reconstruction ${ }^{38}$.

Consequently, a successful surgical approach should be holistic, meaning that both the intra-articular and extra-articular hip pathology should be corrected. Thus, apart from capsular manipulation procedures, labral repair or reconstruction, microfracture and ligamentum teres preservation should be considered when treating hip microinstability.

\section{Conclusion}

In addition to the bony structure, the anatomical integrity of the soft tissues together with their elastic properties, contribute significantly to the stabilization properties of the hip joint. Gymnastics and other related sports can potentially result in significant (acquired) increase in the elasticity of the hip joint capsule and ligaments, and therefore microinstability may become a long-term consequence in those athletes. While the hip joint is a stable joint and does not dislocate with minor forces, microinstability does exist. The alteration of the anatomical relationships of its components, for any reason, such as trauma, systemic disease, dysplastic changes and others may predispose to instability problems and long-term osteoarthritis. Especially, the proximal femoral anatomical abnormalities are thought to contribute significantly to the above concept. Diagnosis of this entity is challenging since symptoms are usually not clear and therefore a high level of suspicion is necessary. When microinstability of the hip has been confirmed, a trial of conservative treatment in preferred at first, leaving the surgical approach for refractory cases. A comprehensive and mindful rehabilitation is vital to achieve satisfactory results.

\section{References}

1. Nepple JJ, Philippon MJ, Campbell KJ, Dornan GJ, Jansson KS, LaPrade RF, Wijdicks CA. The hip fluid seal-Part II: The effect of an acetabular labral tear, repair, resection, and recon- struction on hip stability to distraction. Knee Surg Sports Traumatol Arthrosc. 2014;22:730-736.

2. Philippon MJ, Nepple JJ, Campbell KJ, Dornan GJ, Jansson KS, LaPrade RF, Wijdicks CA. The hip fluid seal-Part I: The effect of an acetabular labral tear, repair, resection, and reconstruction on hip fluid pressurization. Knee Surg Sports Traumatol Arthrosc. 2014;22:722-729.

3. Trindade CA, Sawyer GA, Fukui K, Briggs KK, Philippon MJ. Arthroscopic capsule reconstruction in the hip using iliotibial band allograft. Arthrosc Tech. 2015;4:e71-74.

4. McCormick F, Slikker W 3rd, Harris JD, et al. Evidence of capsular defect following hip arthroscopy. Knee Surg Sports Traumatol Arthrosc. 2014;22:902-905.

5. Shu B, Safran MR. Hip instability: anatomic and clinical considerations of traumatic and atraumatic instability. Clin Sports Med. 2011;30:349-367.

6. Kalisvaart MM, Safran MR. Microinstability of the hip-it does exist: etiology, diagnosis and treatment. J Hip Preserv Surg. 2015;2:123-135.

7. Boykin RE, Anz AW, Bushnell BD, Kocher MS, Stubbs AJ, Philippon MJ. Hip instability. J Am Acad Orthop Surg. 2011;19:340-349.

8. Padulo J, Oliva F, Frizziero A, Maffulli N. Muscles, Ligaments and Tendons Journal. Basic principles and recommendations in clinical and field science research: 2016 Update. MLTJ. 2016;6(1):1-5.

9. Kohnlein, W, Ganz R, Impellizzeri FM, Leunig M. Acetabular morphology: implications for joint-preserving surgery. Clin Orthop Relat Res. 2009;467:682-691.

10. Philippon MJ, Michalski MP, Campbell KJ, Goldsmith MT, Devitt BM, Wijdicks CA, LaPrade RF. An anatomical study of the acetabulum with clinical applications to hip arthroscopy. J Bone Joint Surg. 2014;96:1673-1682.

11. Seldes RM, Tan V, Junt J, Katz M, Winiarsky R, Fitzgerald RH Jr. Anatomy, histologic features, and vascularity of the adult acetabular labrum. Clin Orthop Relat Res. 2001;382:232-340.

12. Tan V, Seldes RM, Katz MA, Freehand AM, Klimkiewicz JJ. Contribution of acetabular labrum to articulating surface area and femoral head coverage in adult hip joints: an anatomic study in cadavera. Am J Orthop (Belle Mead NJ). 2001;30: 809-812.

13. Ferguson SJ, Bryant JT, Ganz R, Ito K. An in vitro investigation of the acetabular labral seal in hip joint mechanics. J Biomech. 2003;36:171-178.

14. Crawford MJ, Dy CJ, Alexander JW, Thompson M, Schroder SJ, Vega CE, Patel RV, Miller AR, McCarthy JC, Lowe WR, Noble PC. The 2007 Frank Stinchfield Award. The biomechanics of the hip labrum and the stability of the hip. Clin Orthop Relat Res. 2007:465:16-22.

15. Walters BL, Cooper JH, Rodriguez JA. New findings in hip capsular anatomy: dimensions of capsular thickness and pericapsular contributions. Arthroscopy. 2014;30:1235-1245.

16. Weidner J, Buchler L, Beck M. Hip capsule dimensions in patients with femoroacetabular impingement: a pilot study. Clin Orthop Relat Res. 2012;470:3306-3312.

17. Nam D, Osbahr DC, Choi D, Ranawat AS, Kelly BT, Coleman $\mathrm{SH}$. Defining the origins of the iliofemoral, ischiofemoral, and pubofemoral ligaments of the hip capsuloligamentous complex utilizing computer navigation. J HSS. 2011;7:239-243.

18. Martin HD, Savage A, Braly BA, Palmer IJ, Beall DP, Kelly B. The function of the hip capsular ligaments: a quantitative report. Arthroscopy. 2008;24:188-195.

19. Telleria JJ, Lindsey DP, Giori NJ, Safran MR. A quantitative assessment of the insertional footprints of the hip joint capsular ligaments and their spanning fibers for reconstruction. Clin Anat. 2014;27:489-497.

20. Demange MK, Kakuda CMS, Pereira CAM, Sakaki MHA da Mota RF. Influence of the femoral head ligament on hip me- 
chanical function. Acta Ortopédica Brasileira. 2007;15:187190.

21. Tan C, Wong W. Absence of the ligament of head of femur in the human hip joint. Singapore Med J. 1990;31:360-363.

22. Kelly BT, Williams RF, Philippon MJ. Hip arthroscopy: current indications, treatment options, and management issues. Am J Sports Med. 2003;31:1020-1037.

23. Bardakos $\mathrm{N}$, Villar $\mathrm{R}$. The ligamentum teres of the adult hip. J Bone Joint Surg. Br. 2009;91:8-15.

24. Martin RL, Kivlan BR, Clemente FR. A cadaveric model for ligamentum teres function: a pilot study. Knee Surg Sports Traumatol Arthrosc. 2013;21:1689-1693.

25. Kivlan BR, Clemente FR, Martin RL, Martin HD. Function of the ligamentum teres during multi-planar movement of the hip joint. Knee Surg Sports Traumatol Arthrosc. 2013;21:16641668.

26. Philippon MJ, Rasmussen MT, Turnbull TL, Trindade CA, Hamming MG, Ellman MB, Harris M, LaPrade RF, Wijdicks $\mathrm{CA}$. Structural properties of the native ligamentum teres. Orthop J Sports Med. 2014.

27. Chen HH, Li AF, Li KC, Wu JJ, Chen TS, Lee MC. Adaptations of ligamentum teres in ischemic necrosis of human femoral head. Clin Orthop Rel Res. 1996;328:268-275.

28. Byrd JT. Hip arthroscopy: the supine position. Clin Sports Med. 2001;20:703-732.

29. Martin HD, Hatem MA, Kivlan BR, Martin LR. Function of the ligamentum teres in limiting hip rotation: a cadaveric tudy. Arthroscopy. 2014;30:1085-1091.

30. Shindle MK, Ranawat AS, Kelly BT. Diagnosis and management of traumatic and atraumatic hip instability in the athletic patient. Clin Sports Med. 2006;25:309-326.

31. Babst D, Steppacher SD, Ganz R, Siebenrock KA, Tannast M. The iliocapsularis muscle: an important stabilizer in the dysplastic hip. Clin Orthop Rel Res. 2011; 469:1728-1734.

32. Scopp JM. Moorman CT 3rd. The assessment of athletic hip injury. Clin Sports Med. 2001;20:647-659.

33. Beighton P. Hypermobility scoring. Br J Rheumatol. 1988; 27:163.

34. Domb BG, Philippon MJ, Giordano BD. Arthroscopic capsulotomy, capsular repair, and capsular plication of the hip: relation to atraumatic instability. Arthroscopy. 2013;29:162-173.

35. Martin HD, Kelly BT, Leunig M, Philippon MJ, Clohisy JC, Martin RL, Sekiya JK, Pietrobon R, Mohtadi NG, Sampson TG, Safran MR. The pattern and technique in the clinical evaluation of the adult hip: the common physical examination tests of hip specialists. Arthroscopy. 2010;26:161-172.

36. Prather H, Harris-Hayes M, Hunt DM, Steger-May K, Mathew $\mathrm{V}$, Clohisy JC. Reliability and agreement of hip range of motion and provocative physical examination tests in asymptomatic volunteers. PM R. 2010;2:p.888-895.

37. Philippon MJ, Ferro FP, Nepple JJ. Hip capsulolabral spacer placement for the treatment of severe capsulolabral adhesions after hip arthroscopy. Arthrosc Tech. 2014;3: e289-e292.

38. Skendzel JG, Goljan P, Philippon MJ. Update on Hip Instability. Minerva Ortopedica e Traumatologica. 2013;64:1-13.

39. Tannast M, Siebenrock KA. Conventional radiographs to assess femoroacetabular impingement. Instr Course Lect. 2009 58:203-212.

40. Mitchell RJ, Gerrie BJ, McCulloch PC, Murphy AJ, Varner KE, Lintner DM, Harris JD. Radiographic Evidence of Hip Microinstability in Elite Ballet. Arthroscopy. 2016;32:1038-1044.

41. Harris JD, Gerrie BJ, Linter DM, Varner KE, McCulloch PC. Microinstability of the hip and the splits radiograph. Orthopedics. 2016;39:e169-75.

42. Mintz DN, Hooper T, Connell D, Buly R, Padget DE, Potter HG Magnetic resonance imaging of the hip: detection of labral and chondral abnormalities using noncontrast imaging. Arthroscopy. 2005;21:385-393.

43. Smith TO, Hilton G, Toms AP, Donell ST, Hing CB. The diagnostic accuracy of acetabular labral tears using magnetic resonance imaging and magnetic resonance arthrography: a meta-analysis. Eur Radiol. 2011;21:863-874.

44. Magerkurth O, Jacobson JA, Morag Y, Caoili E, Fessel D, Sekiya JK. Capsular laxity of the hip: findings at magnetic resonance arthrography. Arthroscopy. 2013;29:1615-1622.

45. Smith MV Sekiya JK. Hip instability. Sports Med Arthrosc. 2010;18:108-112.

46. Philippon MJ. The role of arthroscopic thermal capsulorrhaphy in the hip. Clin Sports Med. 2001;20:817-830.

47. Millis M, Poss R, Murphy S. Osteotomies of the hip in the prevention and treatment of osteoarthritis. Instr Course Lect. 1991;41:145-154.

48. Ganz R, Klaue K, Vinh TS, Mast JW. A new periacetabular osteotomy for the treatment of hip dysplasia. Technique and preliminary results. Clin Orthop Rel Res. 1988;232:26-36.

49. Fukui K, Trindade CA, Briggs KK, Philippon MJ. Arthroscopy of the hip for patients with mild to moderate developmental dysplasia of the hip and femoroacetabular impingement: Outcomes following hip arthroscopy for treatment of chondrolabral damage. Bone Joint J. 2015;97-B:1316-1321.

50. Yamamoto $Y$, Ide T, Nakamura M, Hamada Y, Usui I. Arthroscopic partial limbectomy in hip joints with acetabular hypoplasia. Arthroscopy. 2005;21:586-591.

51. Epstein DM, Rose DJ, Philippon MJ. Arthroscopic management of recurrent low-energy anterior hip dislocation in a dancer. A case report and review of literature. Am J Sports Med. 2010;38:1250-1254. 